\title{
Die Höhenlage von Solifluktionsformen und der Schneegrenze in Graubünden ${ }^{1}$
}

\author{
Gerhard Furrer und Peter Fitze \\ Geographisches Institut der Universität Zürich
}

\begin{abstract}
Grundlage zu dieser Publikation bilden neben den Kartierungen der Verfasser Beobachtungen von Kurt Graf und Ralf Freund. Für die Beratung in methodischen Fragen der Datenverarbeitung danken wir Guido Dorigo. Fräulein Daisy-Claire Brenner, Jürg Bühler und Dieter Wegmann gebührt unser Dank für die Datenübertragung in den Informationsraster. Alle Genannten sind am Geographischen Institut der Universität Zürich tätig.
\end{abstract}

\section{Problemstellung}

In der vorliegenden Arbeit geht es darum, das in den vergangenen Jahren zusammengetragene Beobachtungsmaterial über die Höhenlage der in unseren Alpen am häufigsten vertretenen Typen der Solifluktionsformen nach statistischen Gesichtspunkten zu verarbeiten. Wir möchten auf diesem Wege mit einem Modell Auskunft erhalten über die Gliederung der Hochgebirgsstufe. Bei den hierzu verarbeiteten Formen handelt es sich um solche der ungebundenen (freien) Solifluktion in der Frostschuttstufe. Dies sind einerseits als Vertreter der Großformen die Strukturböden horizontaler oder geneigter Flächen (Hangneigung bis gegen $30^{\circ}$ ), anderseits als Vertreter der Miniaturformen die Erdstreifenböden. Außerdem kommt das Hauptverbreitungsgebiet der Girlanden in der Mattenstufe (alpiner Rasen) zur Darstellung. Bei diesem Formtyp handelt es sich um einen Vertreter der (durch die Vegetation) gebundenen Solifluktionsformen. Um den Verlauf ihrer Untergrenze mit der Waldgrenze vergleichen zu können, wird die Waldgrenze erarbeitet. Diesen beiden zuletzt genannten Grenzflächen kommt in der Geographie große Bedeutung zu, dienen uns doch diese beiden zur Abgrenzung der Hochgebirgslandschaft, um die sich die IGU-Commission on High-Altitude Geoecology bemüht (Troll 1955, Furrer und Fitze 1970).

\section{Die Verarbeitung des kartierten Formenschatzes}

Als Arbeitsgrundlage diente die Landeskarte der Schweiz $(1: 100000)$, auf der alle beobachteten Standorte der einzelnen Formtypen eingetragen wurden. Auf Hängen, wo der gleiche Formtyp durchgehend über eine größere Vertikaldistanz auf- tritt und somit große Flächen mustert, wurde wie folgt kartiert:

Bei einer durchgehenden Vertikalerstreckung bis zu $30 \mathrm{~m}$ berücksichtigten wir den Wert der mittleren Höhenlage (= 1 Punkt), bei $30-60 \mathrm{~m}$ die höchste und tiefste Höhenlage ( $=2$ Punkte) und bei über $60 \mathrm{~m}$ sowohl den Mittelwert als auch den höchsten und tiefsten Wert (= 3 Punkte). Auf diese Art konnte eine gewisse Gewichtung der einzelnen Fundstellen erreicht werden. Die totale Anzahl der Punkte betrug bei den Großformen der Strukturböden 169, bei den Miniaturformen 143 und bei den Girlanden 317.

Über die Landeskarte 1:100 000 wurde anschlieBend ein Raster mit den Massen 1,25 cm (NordSüd) und $1,0 \mathrm{~cm}$ (West-Ost) gelegt (Dieses $5: 4$ Verhältnis entspricht jenem einer Einheit des Schnelldruckers (bei kleinem Zeilenvorschub) und erlaubt somit ein späteres verzerrungsfreies Ausdrucken der Karten.). Anschließend konnte jeder Punkt nach den Koordinaten des Rasters ( $\mathrm{x}$ und $\mathrm{y}$ ) und der Höhenlage (z) gelocht werden.

Soweit die Vorarbeit; die eigentliche Auswertung durch den Computer (IBM 360) setzte sich aus zwei Teilen zusammen:

In einem ersten Schritt wurde der Kanton Graubünden in 13 Regionen (17 bei Girlanden) unterteilt. Dann errechneten wir die Mittelwerte $\overline{(x}, \bar{y}, \bar{z})$ und die Standardabweichung vom Mittelwert der Höhenlage $\left(s_{z}\right)$ (getrennt nach Formtypen) für jede Region. Mit Hilfe des Mittelwertes $\bar{z}$ und der Standardabweichung $s_{z}$ können wir auf die Höhenausdehnung der Kernzone (= Hauptverbreitungsgebiet) und deren Untergrenze schließen, wie im folgenden dargelegt werden soll.

Die Annahme, daß die vertikale Verteilung der beobachteten Formtypen am ehesten einer Normalverteilung entspricht, läßt sich damit stützen, daß in tieferen Lagen die Vegetation und in höheren Lagen perennierende Schneeflecken als begrenzende Faktoren eine immer stärkere Wirkung ausüben. Hinweise für Normalverteilungen von Solifluktionserscheinungen finden wir bei Fitze (1969) und Dorigo (1969). Bei einer normalverteilten Form läßt sich somit die Kernzone (K) mit dem Mittelwert der Höhenlage $\overline{(z)}$ und der Standardabweichung $\left(s_{z}\right)$ definieren: $\mathrm{K}=\overline{\mathrm{z}} \pm \mathrm{s}_{\mathrm{z}}$. 


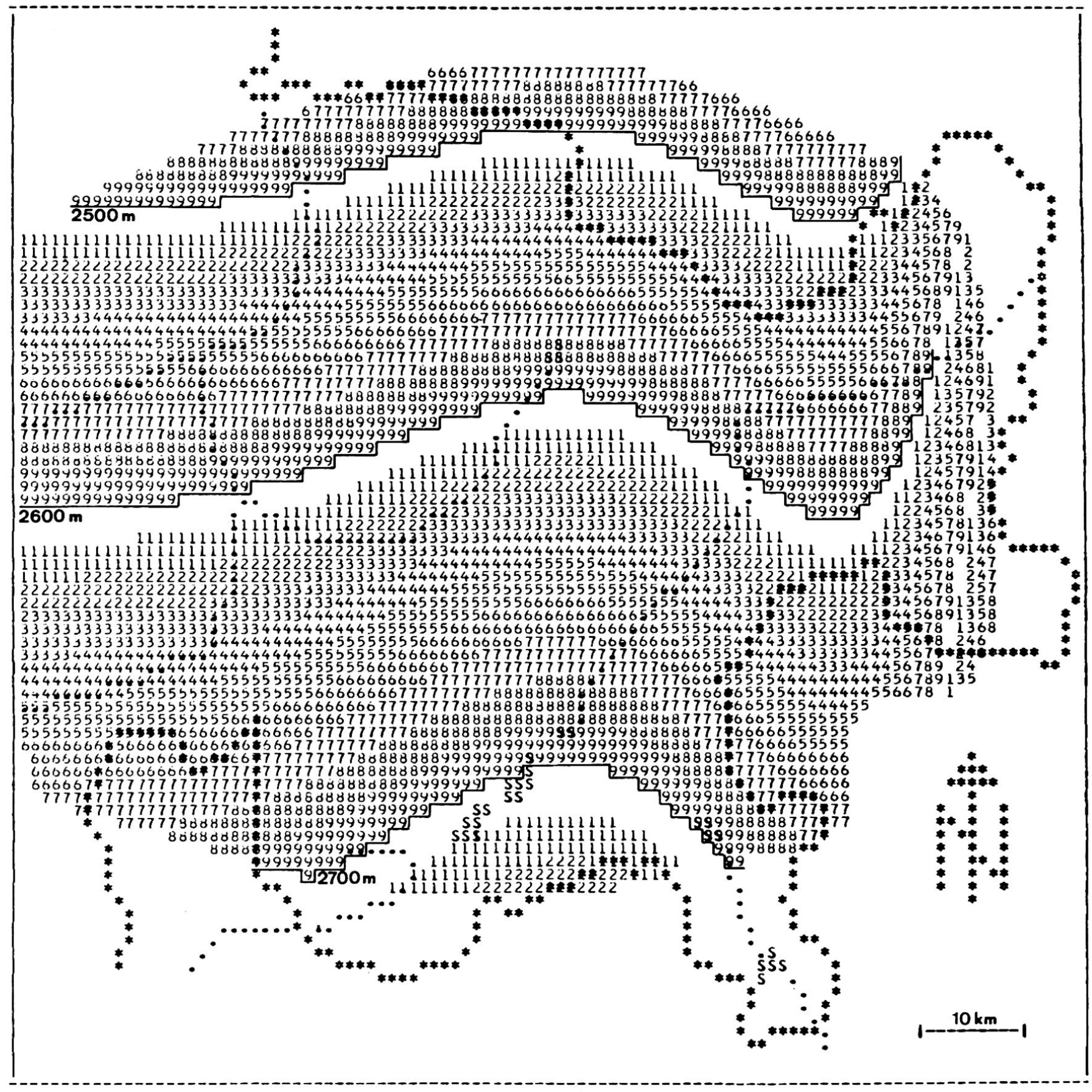

Figur 1. Höhenlage der Kernzonenuntergrenze von Strukturböden (Großformen) im Kanton Graubünden

Innerhalb dieser Kernzone liegen rund zwei Drittel aller Vorkommen, und ihre Untergrenze wird durch den Wert $\bar{z}-S_{\mathbf{z}}$ gebildet.

Alle Berechnungen des zweiten Teils basieren nur noch auf den Mittelwerten und den Untergrenzen der einzelnen Regionen. Für die Karten der Mittelwerte wäre eine Einteilung nach Regionen überflüssig, falls sich die beobachteten Formen gleichmäßig über das ganze Gebiet verteilten. In unserem Falle könnte aber eine sehr dicht kartierte Region mit ihren vielen Beobachtungen eine andere, spärlich kartierte Region noch beeinflussen, was ja vermieden werden soll.
In diesem zweiten Teil wurden mit den Mittelwerten und den Untergrenzen durch schrittweise Regression jene Oberfläche berechnet, die diese Werte am besten annähert. Zur Verfügung standen 70 unabhängige Variablen, alles positive und negative Potenzen von $\mathrm{x}$ und y sowie deren Produkte. In jedem Schritt wurde dabei die Regressionsgleichung um eine Variable erweitert, und zwar immer um jene, die eine optimale Verbesserung der Regression mit Bezug auf die eingegebenen Werte bewirkte. Schrittweise Regressionen wurden durchgeführt für Mittelwerte und Untergrenze jedes Formtyps. Für alle Probleme wurden anschließend die Karten der verschiedenen Regressionsgleichungen ausge- 


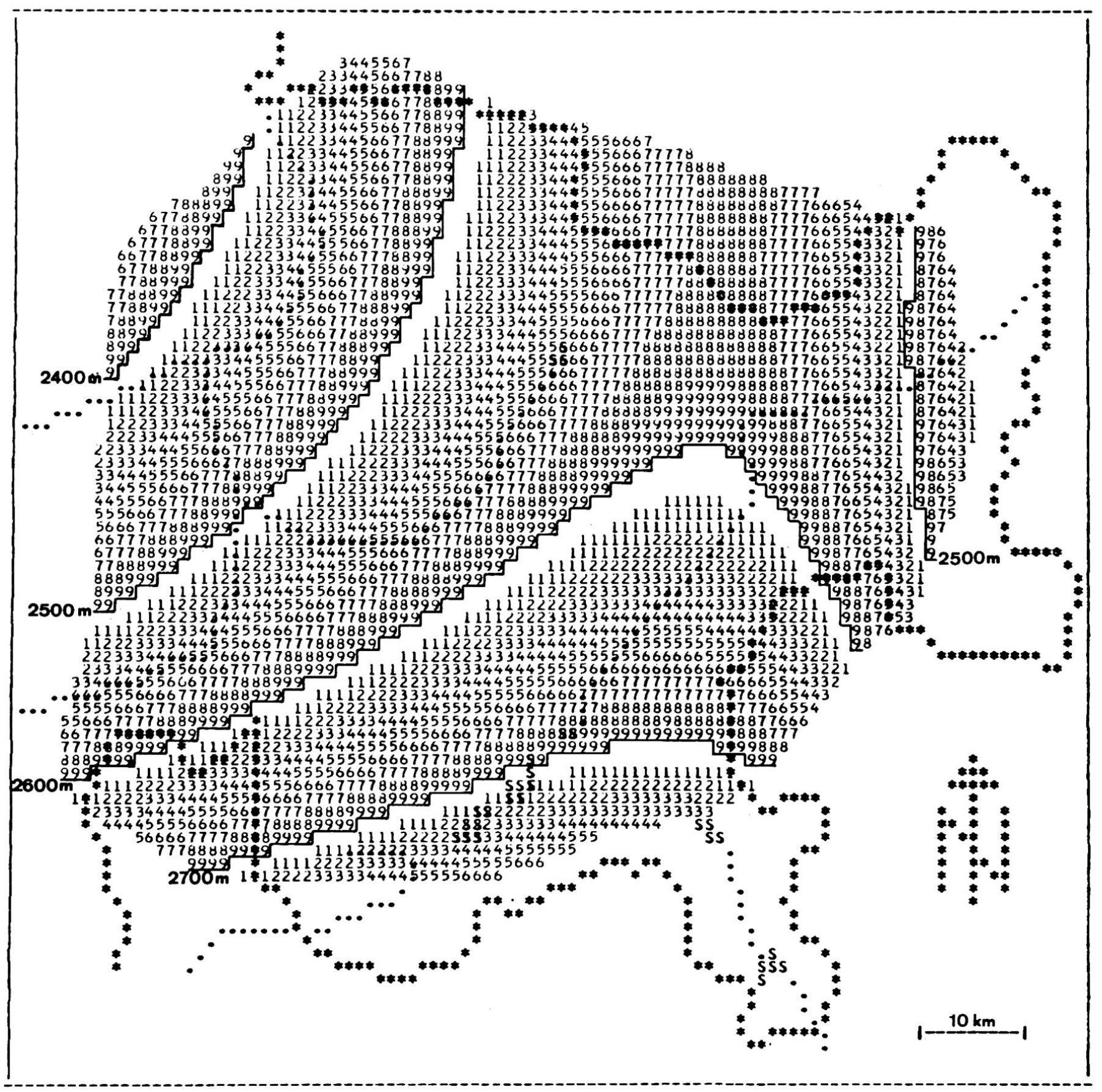

Figur 2. Höhenlage der Kernzonenuntergrenze von Erdstreifen (Miniaturform) im Kanton Graubünden

druckt und jene für den endgültigen Druck ausgewählt, die eine möglichst hohe Korrelation zu den eingegebenen Werten aufwies, aber dennoch ein für das Auge vernünftiges Kartenbild ergab. In allen Karten der Strukturböden (Groß- und Miniaturformen) liegt der multiple Korrelationskoeffizient zwischen 0,6 und 0,8 , bei Girlanden um 0,9; dies bedeutet, daß 40-80\% der Abweichungen vom jeweiligen Mittelwert durch die Regressionsgleichung erklärt sind.

Die Landešarte 1:50 000 diente zur Erarbeitung der Waldgrenze. Rasterfeldern von $4 \mathrm{~km}^{2}$ wurden die beiden folgenden Daten entnommen: höchste Lage von geschlossenem und offenem Wald. Die
Karte basiert auf einer Regression, durchgeführt mit dem höchsten Wert von offenem und geschlossenem Wald in jedem Rasterfeld.

\section{Ergebnisse}

Die Karten der Figuren 1-4 sowie das Profil (Fig. 5) entlang dem Längenkreis $9^{\circ} 42^{\prime} \mathrm{E}$ geben die Höhenverbreitung der untersuchten Formen bzw. den Kernzonenverlauf vom Rhätikon zur Bernina wieder. Daraus ergibt sich eine mögliche Gliederung der Hochgebirgsstufe, wie dies in Figur 6 dargestellt ist. 


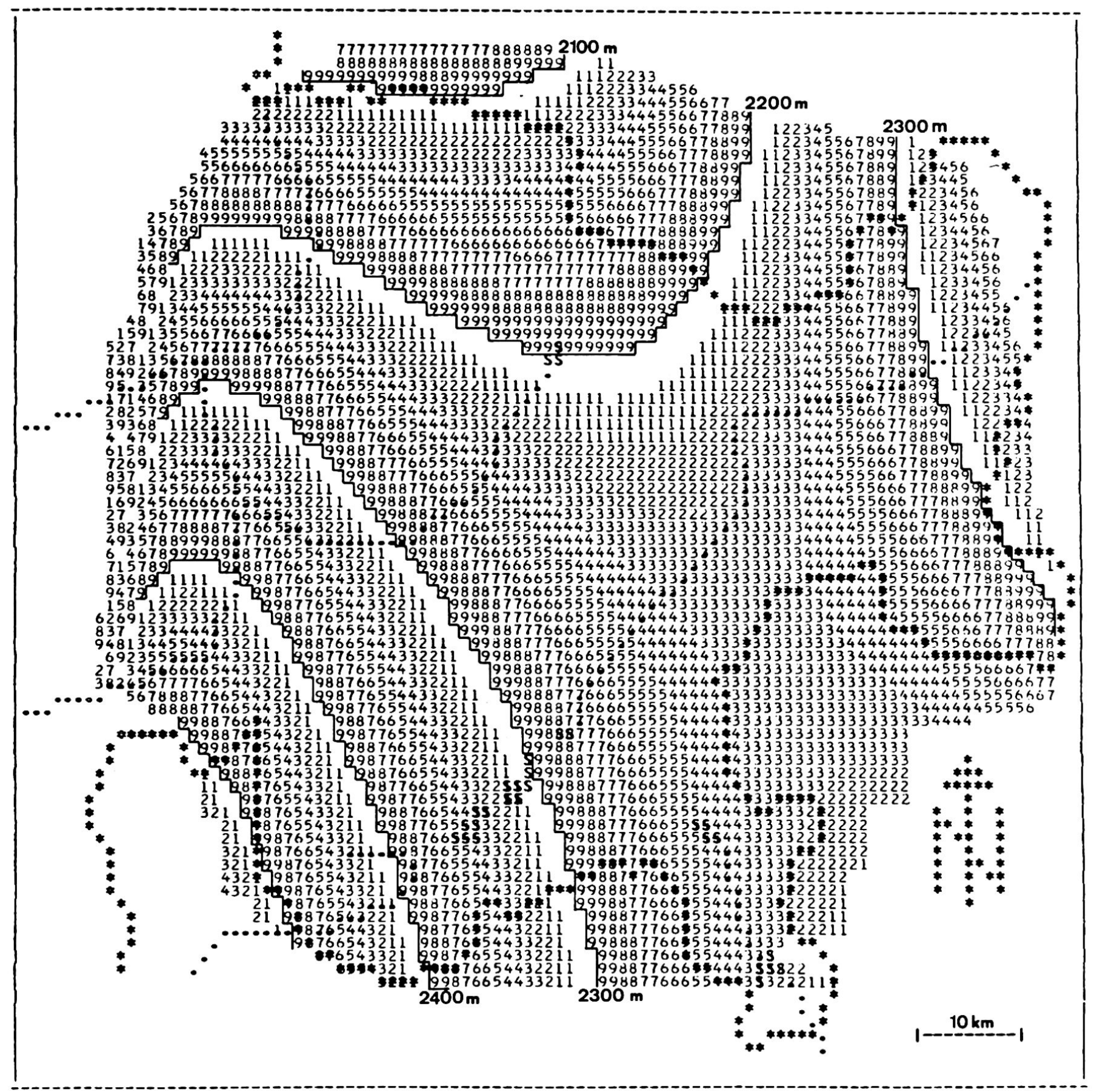

Figur 3. Höhenlage der Kernzonenuntergrenze von Girlanden im Kanton Graubünden

Im Hochgebirge wirkt die Solifluktion formbildend auf Flächen bis rund $30^{\circ}$ Neigung. Selbstverständlich ist diese dort nicht der einzige reliefbildende Vorgang. Die von ihr geschaffenen Kleinformen aber sowie Solifluktionsdecken, die sich an ihrer Oberfläche heute noch bewegen, gehören zum charakteristischen Formenschatz der Hochgebirgsstufe. Sie können daher zur Abgrenzung dieser Stufe und $\mathrm{zu}$ ihrer Unterteilung herangezogen werden (Troll 1955, Furrer und Fitze 1970).

Einmal kann durch Scheidung der Verbreitungsgebiete von gebundenen und ungebundenen Solifluktionsfarmen die Frostschutt- von der Matten- stufe getrennt werden. Wo die Solifluktion als formbildender Vorgang auf größeren Flächen zurücktritt, legen wir die Untergrenze der subnivalen Höhenstufe fest. Diese dient gleichzeitig zur Abgrenzung der Hochgebirgsstufe. In Figur 6 ist hierzu lediglich die Untergrenze der Girlandenkernzone benützt worden. Um die Untergrenze des Hauptverbreitungsgebietes der Solifluktionsformen noch exakter festlegen zu können, bieten sich außerdem die Wanderblöcke an, jene Blöcke, die in der Mattenstufe mehrere Meter weit talwärts gleiten, vor sich die Vegetation aufstauchen und hinter sich eine Schleifspur zurücklassen. 


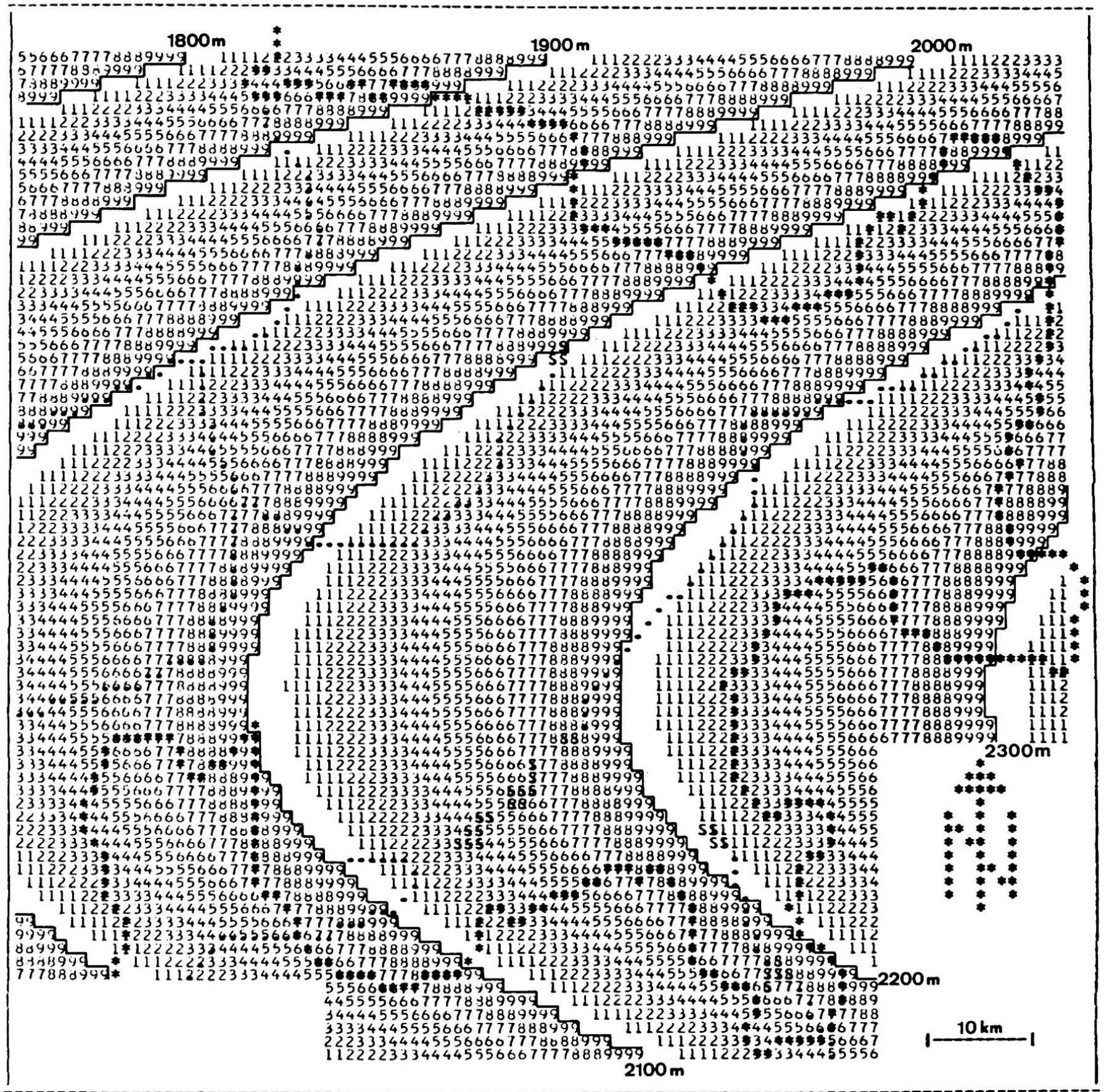

Figur 4. Höhenlage der Waldgrenze im Kanton Graubünden

Die Darstellung in Figur 6 basiert auf den errechneten Werten aus dem gesamten Kanton Graubünden, während die Karten und das Profil (Fig. 5) auf regional zusammengefaßten und verarbeiteten Beobachtungen beruhen. Daher ist es verständlich, daß die Bandbreiten der Kernzonen des bearbeiteten Formenschatzes in Figur 5 in ihrer Ausdehnung schwanken. Die errechneten vertikalen Ausdehnungen der Kernzonen für Graubünden gehen aus folgender Zusammenstellung hervor:
Berechnete vertikale

Mittlere Höhenlage Ausdehnung der Kernzone der Kernzone

$\left(2 \cdot s_{z}\right)$

$290 \mathrm{~m}$ (169 Beobachtungen)

$340 \mathrm{~m}$ (143 Beobachtungen)

$370 \mathrm{~m}$ (317 Beobachtungen)

$2640 \mathrm{~m}$ $2420 \mathrm{~m}$
Durch Kartierungen ermittelte Kernzonenausdehnung van früheren Beobachtungen (Furrer 1965)

gut $200 \mathrm{~m}$ $230 \mathrm{~m}$ um $350 \mathrm{~m}$ 


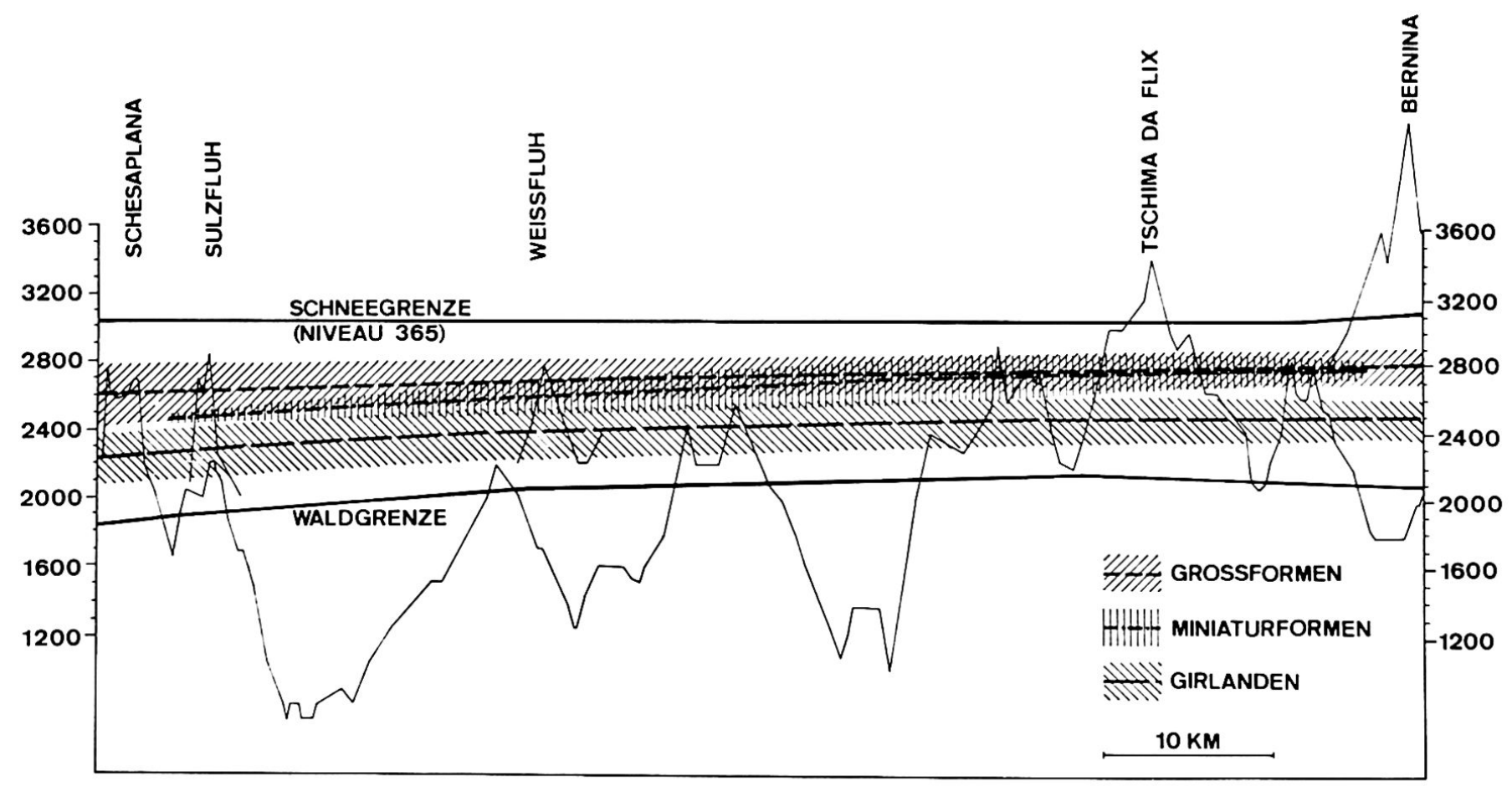

Figur 5. Verlauf von drei Kernzonen und deren mittlerer Höhenlage in nord-südlicher Richtung durch Graubünden

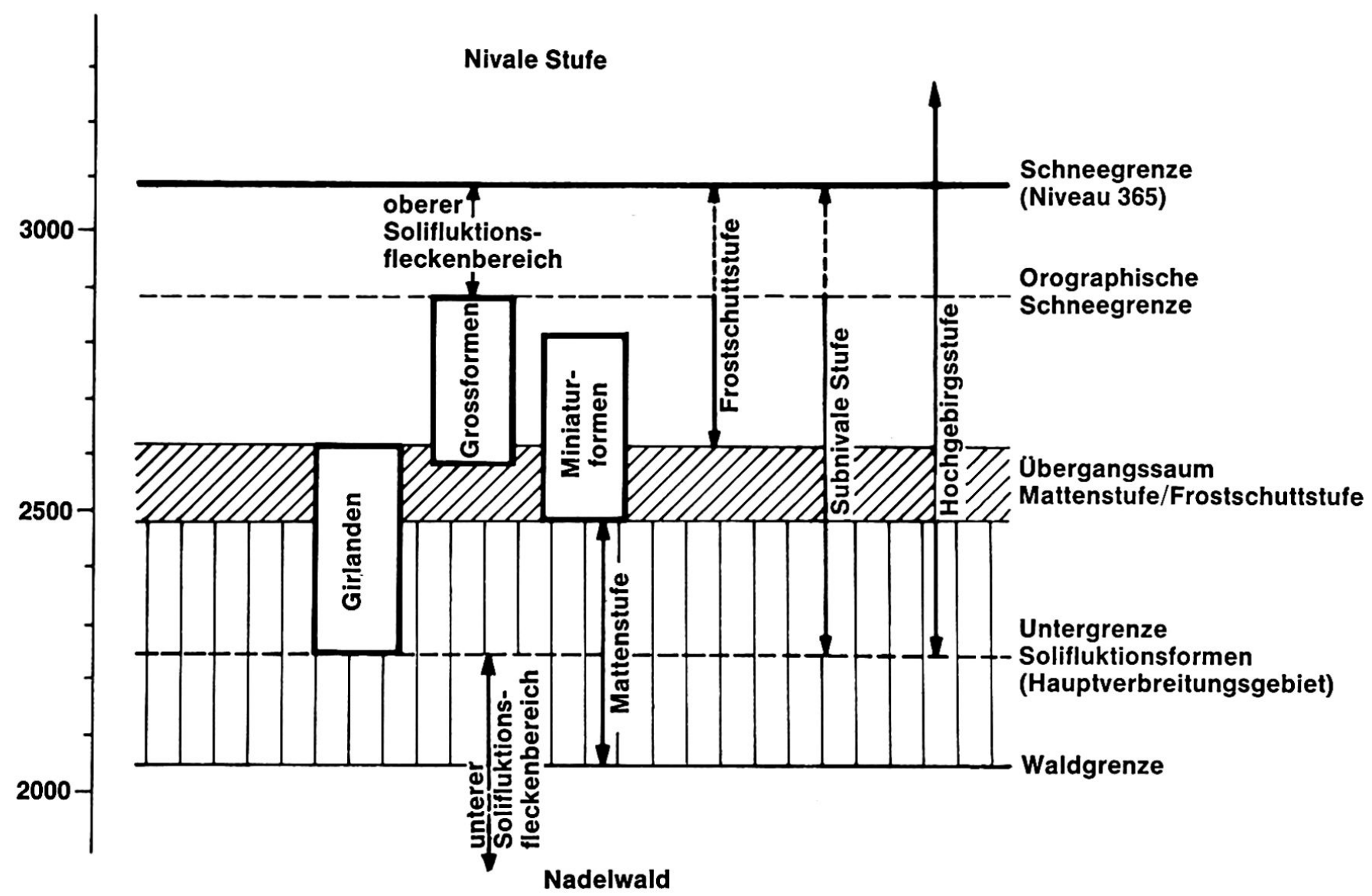

Figur 6. Abgrenzung und Gliederung der Hochgebirgsstufe mit Hilfe van Solifluktionsformen am Beispiel des Kantons Graubünden 
Nach oben wird die Frostschuttstufe durch die Schneegrenze abgegrenzt. Diese ökologisch wichtige Linie ist in der Natur als Grenzsaum ausgebildet. Gerade wegen ihrer Bedeutung für die Lebewesen, die Bodenbildung und die reliefgestaltenden Vorgänge befriedigen alle jene Festlegungen der Schneegrenze nicht, die sich auf Beobachtungen an Gletschern oder Berechnungen mit Hilfe horizontaler Beobachtungsfelder (klimatische Schneegrenze, Niveau 365, vgl. Escher 1970) stützen. Den Geographen interessiert eine Schneegrenze, welche auf das Relief Rücksicht nimmt und den tatsächlichen Verhältnissen Rechnung trägt. Diese «orographische Schneegrenze» im Mittel vieler Jahre zu erfassen, erscheint als fast unlösbares Problem.

Um eine solche anzunähern, bieten sich die Solifluktionsformen, wenigstens für Flächen bis rund $30^{\circ}$ Neigung, an. Feldbeobachtungen haben ergeben, daß die höchstgelegenen Strukturböden auf horizontalen wie geneigten Flächen bis nahe an das Niveau 365 auftreten: So liegen beispielsweise im Raume Zermatt - wo nach Escher (1970) die klimatische Schneegrenze in einer Höhe von $3450 \mathrm{~m}$ liegt - die obersten Vertreter der Großformen auf $3400 \mathrm{~m}$ (Furrer 1965). Wegen ihrer Seltenheit wird es uns aber nur in Glücksfällen gelingen, mit ihrer Hilfe die klimatische Schneegrenze (als Niveau 365) zu bestimmen. Da wir außerdem nicht nach einer klimatischen, sondern nach einer orographischen fragen, scheint ein anderer Weg angezeigt. Besonders die obersten Vertreter der Großformen von Strukturböden liegen auf schneefreien Flächen in der hoch- bis spätsommerlichen Schneefleckenregion. Da sich diese Formen nicht in wenigen Sommern zur vollen Reife entwickeln, ist anzunehmen, daß ihre Standorte in der Regel jedes Jahr schneefrei werden. Diese Schneefleckenregion des Hochsommers läßt sich talwärts annähernd durch die Obergrenze des Hauptverbreitungsgebietes (Kernzone) der Großformen unserer Strukturböden abgrenzen. Damit gelangen wir zu einer Angabe über die Höhenlage der Schneegrenze, welche - zumindest auf Flächen bis $30^{\circ}$ Neigung - der orographischen oder lokalen nahe kommt. Eine solche Schneegrenze soll im Sinne von Ratzel (1889) verstanden sein als «jene Linie, welche die untern Ränder der dauernden Schneeflecken verbindet». Sie liegt, wie Figur 6 belegt, $200 \mathrm{~m}$ unter dem Niveau
365. So ersetzen Kartierungen von Strukturböden, im Hochsommer durchgeführt, mehrjährige Schneegrenzbeobachtungen, die zudem von der methodischen Seite her schwierig durchzuführen sind.

\section{Anmerkung}

1 Die Feldarbeit wurde mittels Unterstützung der Stiftung für wissenschaftliche Forschung an der Universität Zürich ermöglicht.

\section{Literatur}

Dorigo G.: Untersuchungen zur (gebundenen) Solifluktion am Sustenpa $\beta$ und auf der Melchsee-Frutt. Dipl. arb. Univ. Zürich, 1969.

Escher H.: Die Bestimmung der klimatischen Schneegrenze in den Schweizer Alpen. Geogr.Helv. 1, 1970.

Fitze P.: Untersuchungen von Solifluktionserscheinungen im Alpenquerprofil zwischen Säntis und Lago di Como. Diss. Univ. Zürich, 1969.

Furrer G.: Die Höhenlage von subnivalen Bodenformen. Habilitationsschrift Univ. Zürich, 1965. Furrer G. und Fitze P.: Die Hochgebirgsstufe - ihre Abgrenzung mit Hilfe der Solifluktionsgrenze. Geogr. Helv. 4, 1970.

Ratzel F.: Höhengrenzen und Höhengürtel. Z. dt.österreich. Alpenver., Bd. 10, 1889.

Troll C.: UUber das Wesen der Hochgebirgsnatur. Jahrbuch österreich. Alpenver., 1955.

\section{Résumé}

A l'aide de formes de solifluxion on essaie à fixer les limites de la zone de la haute montagne et à la diviser en «Frostschuttzone» et "Mattenstufe». La limite supérieure de la zone principale de sols structurés peut être employée pour trouver la ligne des neiges orographique. Celle-ci se trouve dans les Grisons $200 \mathrm{~m}$ en dessous de la ligne des neiges climatique. 